\title{
Intra-Positional and Inter-Positional Differences in Somatotype Components and Proportions of Particular Somatotype Categories in Youth Volleyball Players
}

\author{
${ }^{1}$ Zoran Grgantov, ${ }^{1,2,3}$ Johnny Padulo, ${ }^{1}$ Mirjana Milić, ${ }^{4}$ Luca Paolo Ardigò*, ${ }^{1}$ Marko Erceg, \\ ${ }^{1}$ Drazen Cular
}

${ }^{1}$ Faculty of Kinesiology, University of Split, Split, Croatia. ${ }^{2}$ University eCampus, Novedrate, Italy. ${ }^{3}$ Tunisian Research Laboratory "Sports Performance Optimization", National Center of Medicine and Science in Sport, Tunis, Tunisia. ${ }^{4}$ School of Exercise and Sport Science, Department of Neurosciences, Biomedicine and Movement Sciences, University of Verona, Verona, Italy.

\begin{abstract}
Objective(s). Main aim of this cross-sectional study was to analyse intra-positional, inter-positional differences in proportions of particular somatotype categories in youth volleyball players. Methods. Heath-Carter method was used to determine somatotype characteristics of 181 young female volleyball players (age 14.05 \pm 0.93 , height $170.03 \pm 7.61$ $\mathrm{cm}$, mass $57.80 \pm 8.59 \mathrm{~kg}$, body mass index $19.99 \pm 2.37 \mathrm{~kg} / \mathrm{m}^{2}$, somatotype 4.33-2.79-3.73 $\pm 1.02-1.02-1.25$ ). Dependent variables were playing position, player efficacy. Only 7 somatotype categories (of possible 13) were obtained. Results. By analysing intra-positional differences, a significantly higher frequency of somatotype categories dominated by the ectomorph component was established in a subsample of more successful players, and a significantly higher frequency of somatotype categories dominated by the endomorph component was found in a subsample of less efficient players. The most frequent somatotype category in all player positions in a sample of more efficient volleyball players is the balanced ectomorph. The smallest number of somatotype categories (only three) was obtained in the subsample of more efficient middle hitters. It indirectly indicates that the most stringent requirements for selection according to the body build are for that position. Similar small inter-positional differences were obtained in both subsamples. Ectomorph somatotype component was most pronounced in young middle-hitters, and endomorph in liberos. Small inter-positional differences in both subsamples are probably consequences of previous selection processes within the clubs. Conclusion. Results obtained in this study provide a clearer insight into inter-positional, intra-positional differences in somatotype categories of young female volleyball players.
\end{abstract}

KEY WORDS: Female Volleyball, U15 Age Group, Somatotyping, Player Position, Player Efficacy.

\section{INTRODUCTION}

Somatotyping is one of the most frequently used techniques for analysing the body build. It is usually applied in volleyball to determine the body build specificities of male and female volleyball players of different age groups (1-16).

\footnotetext{
*. Corresponding Author:

Luca Paolo Ardigò

E-mail: luca.ardigo@univr.it
} 
It is well known that the lack of appropriate body build might result in poor performance in top-level volleyball. Although some of these characteristics can be improved through training, the basic ones required for the sport of volleyball may be essentially inherited (e.g., body height and length of limbs). It is of paramount importance for coaches to understand the significance of taking into account these basic body characteristics for identification of talented young players. Inappropriate identification of young female players without considering anthropometric features could become an obstacle for future developments to become toplevel players (17).

In team sports, the specificities of each player position must be taken into consideration in that process. In volleyball, middle hitters, passerhitters, opposites, setters and liberos differ in their roles and require different skills and tactics during matches. Therefore, differences are also expected in their somatotype components.

It is therefore surprising that in already relatively rare studies on young female volleyball players $(1,18-21)$ inter-positional differences in somatotype have not been investigated. Studies in which those differences were analysed on a sample of senior female volleyball players are rare $(17,22,23)$. In our previous study (12) two young national team members who play at libero and middle blocker position were selected from the overall sample. Obvious differences in their somatotypes, despite the same performance level, indirectly pointed to the necessity of further analysis of somatotype components according to positions. It was emphasized in the same study that the calculation of mean values of the somatotype components without individual classification of players into somatotype categories does not allow insight into the somatotype specificities of individual players.

Within a particular sport activity, physique varies according to the performance level as well. The higher the level, the higher the tendency to conform the body characteristics to an ideal model $(2,3,7,24)$.

However, there have not been enough studies investigating whether body build varies according to the performance level in a sample of young female volleyball players as well.
There have been no studies that investigated intra-positional differences according to performance level. Such studies could provide volleyball coaches with useful indications to better detect talents for volleyball and also to assign the most appropriate player positions according to players' body build. In younger age groups it is not enough to assess the performance level of each player by her placement in a competition or by competition rank, as it is frequently done for senior players. Greater differences in individual player quality within a team in younger age groups indicate the necessity of taking into consideration the intrateam differences as well, and not only the interteam differences when evaluating competitive efficacy of each player (12).

Therefore, the aim of this research, which was conducted on a relatively large subject sample, is to analyse intra-positional (according to performance level) and inter-positional differences in somatotype components and proportions of particular somatotype categories in youth volleyball players.

\section{MATERIALS AND METHODS}

Participants. The subject sample consisted of 181 Croatian female volleyball players. Age was $14.05 \pm 0.93$ years, height was $170.03 \pm 7.61$ $\mathrm{cm}$, body mass was $57.80 \pm 8.59 \mathrm{~kg}$, body mass index was $19.99 \pm 2.37 \mathrm{~kg} / \mathrm{m}^{2}$ and somatotype was 4.33-2.79-3.73 $\pm 1.02-1.02-1.25$. All volleyball players in this sample have been involved in the training process for averagely $3.1 \pm 0.7$ years in 17 volleyball clubs of the Dalmatia region. In addition to their weekly 4.56 hour engagement, they also participate in matches of a weekend league for young players (minimum of 22 matches during a season). The number of players was divided according to player positions and the "criterion of efficacy" (i.e., LE-less efficient players or ME-more efficient players). For $\mathrm{LE}$ and $\mathrm{ME}$ we have, respectively, 13 and 17 for setters (age $14.12 \pm 0.87 \mathrm{yrs}), 24$ and 17 for opposites (13.98 $\pm 1.01 \mathrm{yrs}), 22$ and 32 for passer-hitters (14.08 $\pm 0.90 \mathrm{yrs}), 11$ and 17 for middle blockers $(14.01 \pm 0.95 \mathrm{yrs})$, and 16 and 22 for liberos (14.07 $\pm 0.91 \mathrm{yrs}$; totals 86 and 95, 14.05 \pm 0.93 yrs). An informed written consent for participating in the research was given by all 
subjects and their parents. The researchers had full accordance of all volleyball clubs as well as the expert committee of the Volleyball Association of the Dalmatia Region for conducting the research. The local university Ethical Committee verified that this investigation complied with all the ethical standards for scientific investigations involving human participants.

Measures. The present study is a crosssectional study investigating Croatian female youth volleyball players $(n=181)$ who were measured using ten anthropometric measures, which represent independent variables. Sample size determination was not performed according to relevant literature due to the limited availability of the players (25).

Based on the anthropometric characteristics, somatotype components and categories using the Heath-Carter method (26-29) were calculated.

Player position and player efficacy served as dependent variables in this research. Based on their position in the team, female volleyball players were divided into five groups: setters, opposites, passer-hitters, middle blockers and liberos.

Procedures. The following ten anthropometric measures were used in this research: body height $(\mathrm{cm})$ and mass $(\mathrm{kg})$; triceps, subscapular, supraspinale and calf skinfolds $(\mathrm{cm})$; flexed arm and calf girth $(\mathrm{cm})$; humerus and femur breadth $(\mathrm{cm})$, following the guidelines outlined by the International Society for the Advancement of Kinanthropometry (30). The Anthropometer Measuring Set by Martin and Harpenden skinfold caliper (UK) were used. Carter and Heath's equations were applied for the calculation of anthropometric somatotypes (31). BMI was calculated by dividing the weight in kilograms by the square of the height in meters.

Player quality on a five-point Likert scale served as a criterion variable (12,32-34). A grade of 1-5 was assigned to each player based on the following two criteria:

1. Team ranking in the competition: all teams competed in the Croatian regional championship and were classified into 3 categories $\left(1^{\text {th }}-4^{\text {th }}\right.$ place; $5^{\text {th }}-8^{\text {th }}$ place; $9^{\text {th }}-12^{\text {th }}$ place) based on their ranking in the championship.
2. Player quality within the team (as evaluated by the coaches). Each coach divided his/her players into 3 groups (the most efficient players, average - other starters and non-starters who contribute to game quality, the least efficient - players who very rarely or never enter the game).

For instance, for $1^{\text {th }}-4^{\text {th }}$ team ranking in the Croatian regional championship and member of the national team Likert score 5, while for $9^{\text {th }}$ $12^{\text {th }}$ ranking and least efficient players in the team Likert score 1. The less efficient group included all players who were assigned grades of $1-3$, and the group of more efficient players included all players who were assigned grades 4 and 5 .

Measurements were conducted in indoor volleyball gyms during morning hours in July and August of 2012, just after the end of the youth league season and prior to beginning the new cycle of the annual training process.

The measurements were made according to the International Society for the Advancement of Kinanthropometry protocol (35) on the right side of the body, while the left dominant side of the body was measured in nine volleyball players, as was originally described by Carter and Heath (28) and by Duquet and Carter (36) for the purposes of somatotype analysis.

Two to three measurements were taken at each site. The average value was used in any further calculation if two measurements were taken, and the median value was used if three measurements were taken. One assistant recorded the values and helped standardize measurement techniques.

Skinfold sites were measured in succession to avoid experimenter bias (complete variable set was measured before repeating the measurement at the same site for the second and then third time).

Statistical Analysis. Data analysis methods included the calculation of descriptive statistical indicators (mean, median, minimum and maximum results, and standard deviations) of ten morphological measures and 3 somatotype components on the overall sample. These methods were also used on the defined subsamples in relation to player positions and intra-positional values according to the criterion of efficacy. The Kolmogorov-Smirnov test (KS) 
was applied to calculate the maximal difference value for determining the normality of variable distribution. All volleyball players were classified into one of the 13 possible somatotype categories. In such a way, 7 somatotype categories were obtained (31).

The frequency and percentage of each category were calculated within the whole sample, the subsamples of efficient and less efficient players and for all player positions. Analysis of differences in proportions was applied to determine whether there are significant differences between and within the player positions in the frequency of each somatotype category between more and less efficient players.

Somatotype was calculated by using the Somatotype Ver. 1.2.5 software and statistical data analysis was performed by using the Statistica Ver. 11.00 software.

\section{RESULTS}

Table 1 shows descriptive variables of morphological characteristics and somatotype components of youth female volleyball players.

Table 1. Descriptive statistics of variables of morphological characteristics and somatotype components in Croatian young female volleyball players.

\begin{tabular}{lccccc}
\hline \multicolumn{1}{c}{ Variables } & Median & Mean & SD & Range & KS \\
\hline Endomorphy & 4.30 & 4.33 & 1.02 & $1.4-7.7$ & 0.06 \\
Mesomorphy & 2.70 & 2.79 & 1.02 & $0.3-5.7$ & 0.07 \\
Ectomorphy & 3.80 & 3.73 & 1.25 & $0.7-8.5$ & 0.05 \\
Height & 170.10 & 170.03 & 7.61 & $154-193.1$ & 0.05 \\
Mass & 57.00 & 57.80 & 8.59 & $35.7-81.6$ & 0.05 \\
Triceps skinfolds & 16.73 & 17.06 & 4.73 & $6.4-31.53$ & 0.07 \\
Subscapular skinfolds & 9.83 & 10.17 & 2.74 & $4.2-20.13$ & 0.11 \\
Supraspinale skinfolds & 15.27 & 15.82 & 5.04 & $4.83-35.73$ & 0.07 \\
Calf skinfolds & 13.53 & 14.02 & 4.22 & $3.73-25.73$ & 0.07 \\
Arm girth & 26.00 & 25.97 & 2.31 & $16.26-31.4$ & 0.06 \\
Calf girth & 34.20 & 34.12 & 2.43 & $26.5 .7-41$ & 0.06 \\
Humerus breadth & 6.10 & 6.09 & 0.34 & $5.4-7.3$ & 0.09 \\
Femur breadth & 9.20 & 9.16 & 0.44 & $8.1-10.2$ & 0.06 \\
\hline \multicolumn{2}{r}{} & & & & Test= 0.12 \\
\hline
\end{tabular}

SD-standard deviation, KS-Kolmogorov-Smirnov test. $(\mathbf{n = 1 8 1})$.

The analysis of the variables distribution shows that all variables were normally distributed and that there were no deviations from a normal distribution. Normality of distribution was tested by using a KS with a critical value of 0.12 . All somatotype components are averagely expressed (within the range of 2.5 to 5). The endomorph somatotype component is dominant, while the mesomorph component is the least expressed one. Given the values of each somatotype component, youth Croatian female volleyball players averagely fit the ectomorphendomorph somatotype category.
Frequency and percentage of somatotype categories in a whole sample, subsamples of less efficient and more efficient players, as well as differences in proportions between subsamples are shown in Table 2.

In the total sample, as well as in the subsamples of more efficient and less efficient young female volleyball players (YFVP), 7 somatotype categories were found (Table 2). In the total sample the highest percentage of players (about 45\%) fits the 4 somatotype categories that are dominated by the endomorph component. Individually, the most frequent somatotype category is the balanced ectomorph. 
In the subsample of more efficient youth players, almost half of them fit the balanced ectomorph somatotype category, and one fifth of them fit the endomorph-ectomorph category.
A significantly higher proportion of more efficient players in comparison to less efficient players fit the previously mentioned somatotype categories.

Table 2. Frequency and percentage of each somatotype category and significance of differences in proportions between less effcient and more efficient Croatian female volleyball players.

\begin{tabular}{cccccccc}
\hline \multirow{2}{*}{ Somatotype category } & \multicolumn{2}{c}{$\begin{array}{c}\text { Total sample } \\
(\mathrm{n}=181)\end{array}$} & $\begin{array}{c}\text { Less efficient } \\
(\mathrm{n}=85)\end{array}$ & $\begin{array}{c}\text { More efficient } \\
(\mathrm{n}=96)\end{array}$ & $\mathbf{p}$ \\
\cline { 2 - 8 } & $\mathbf{n}$ & $\mathbf{\%}$ & $\mathbf{n}$ & $\mathbf{\%}$ & $\mathbf{n}$ & $\mathbf{\%}$ & $\mathbf{p}$ \\
\hline Endomorph-ectomorph & 27 & 14.92 & 7 & 8.14 & 20 & 20.05 & 0.02 \\
\hline Ectomorphic endomorph & 21 & 11.60 & 15 & 17.44 & 6 & 6.32 & 0.01 \\
\hline Balanced endomorph & 14 & 7.73 & 10 & 11.63 & 4 & 4.21 & 0.03 \\
\hline Mesomorphic endomorph & 40 & 22.10 & 30 & 34.88 & 10 & 10.53 & 0.00 \\
\hline Mesomorph-endomorph & 7 & 3.84 & 6 & 6.98 & 1 & 1.05 & 0.02 \\
\hline Balanced ectomorph & 54 & 29.83 & 9 & 10.47 & 45 & 47.37 & 0.00 \\
\hline
\end{tabular}

$\mathbf{n}$ :subject frequency, $\%$ :relative values, $\mathbf{p}$ :significance of differences in proportions between less efficient and more efficient female volleyball players.

As much as $70 \%$ of less efficient YFVP belongs to the 4 somatotype categories that are characterized by the dominance of the endomorph somatotype component. At the same time, more than one third of them fit the mesomorphic endomorph category. A significantly higher proportion of less efficient YFVP in comparison to more efficient YFVP fits these categories.

As it can be seen in Table 3, 6 somatotype categories (out of the possible 13) were identified at the setter position in both subsamples. A significantly higher proportion of balanced ectomorph and endomorph-ectomorph somatotype categories was identified in the subsample of more efficient YFVP, while a significantly higher proportion of mesomorphic endomorph and mesomorph-endomorph somatotype categories was identified in the subsample of less efficient YFVP in this position.

In opposite hitters, 6 somatotype categories were identified in the subsample of less efficient YFVP, and 5 categories in the subsample of more efficient YFVP.

Withal, the biggest percentage of less efficient opposite hitters fits the mesomorphic endomorph and ectomorphic endomorph categories, and almost one half of more efficient players in this position fit the balanced ectomorph category. A significantly higher proportion of mesomorphic endomorph, and a significantly lower proportion of balanced ectomorph and endomorph-ectomorph somatotype categories were identified in the subsample of less efficient opposite hitters.

Both subsamples of passer-hitters are distributed in six somatotype categories. About 3/4 of more efficient passer-hitters belong to two categories (endomorph-ectomorph and balanced ectomorph). At the same time, less efficient passer-hitters are equally distributed in five somatotype categories with a slight dominance of the mesomorphic endomorph category. A significantly higher proportion of balanced endomorph and ectomorphic endomorph categories can be seen in a subsample of less efficient passer-hitters in comparison with more efficient passer hitters.

In the subsample of more efficient middle hitters only three somatotype categories were obtained (balanced ectomorph, central and endomorph-ectomorph). More than $80 \%$ of them fits the balanced ectomorph category. In less efficient middle hitters, a larger number of somatotype categories (5) can be seen, with dominance of central and ectomorphicendomorph categories. A significantly higher proportion of the balanced ectomorph somatotype category was identified in more efficient middle hitters and a significantly higher proportion of the ectomorphic-endomorph somatotype category was found in less efficient middle hitters. 
Table 3. Descriptive statistics of each somatotype category and significance of intra-positional differences in proportions between less efficient and more efficient Croatian female volleyball players.

\begin{tabular}{|c|c|c|c|c|c|c|c|c|c|c|}
\hline \multirow{3}{*}{ Somatotype category } & \multicolumn{5}{|c|}{ SETTER } & \multicolumn{5}{|c|}{ OPPOSITE } \\
\hline & \multicolumn{2}{|c|}{$\begin{array}{c}\text { Less efficient } \\
n=13\end{array}$} & \multicolumn{2}{|c|}{$\begin{array}{c}\text { More } \\
\text { efficient } \\
n=17\end{array}$} & \multirow[b]{2}{*}{$\mathrm{p}$} & \multicolumn{2}{|c|}{$\begin{array}{c}\text { Less } \\
\text { efficient } \\
n=24\end{array}$} & \multicolumn{3}{|c|}{$\begin{array}{c}\text { More } \\
\text { efficient } \\
\mathrm{n}=17\end{array}$} \\
\hline & $\mathrm{n}$ & $\%$ & $\mathrm{n}$ & $\%$ & & $\mathrm{n}$ & $\%$ & $\mathrm{n}$ & $\%$ & $\mathrm{p}$ \\
\hline Endomorph-ectomorph & 0 & 0.00 & 3 & 17.65 & 0.05 & 0 & 0.00 & 3 & 17.65 & 0.02 \\
\hline Ectomorphic endomorph & 1 & 7.69 & 2 & 11.76 & 0.36 & 5 & 20.83 & 3 & 17.65 & 0.40 \\
\hline Balanced endomorph & 2 & 15.38 & 1 & 5.88 & 0.95 & 3 & 12.50 & 1 & 5.88 & 0.24 \\
\hline Mesomorphic endomorph & 5 & 38.46 & 1 & 5.88 & 0.01 & 10 & 41.67 & 2 & 11.76 & 0.02 \\
\hline Mesomorph-endomorph & 2 & 15,38 & 0 & 0.00 & 0.05 & 1 & 4.17 & 0 & 0.00 & 0.20 \\
\hline Balanced ectomorph & 1 & 7.69 & 8 & 47.06 & 0.01 & 2 & 8.33 & 8 & 47.06 & 0.00 \\
\hline Central & 2 & 15.38 & 2 & 11.76 & 0.37 & 3 & 12.50 & 0 & 0.00 & 0.07 \\
\hline \multirow{3}{*}{ Somatotype category } & \multicolumn{4}{|c|}{ PASSER-HITTER } & & \multicolumn{5}{|c|}{ MIDDLE BLOCKER } \\
\hline & \multicolumn{2}{|c|}{$\begin{array}{l}\begin{array}{c}\text { Less efficient } \\
\mathrm{n}=22\end{array} \\
\end{array}$} & \multicolumn{2}{|c|}{$\begin{array}{c}\text { More } \\
\text { efficient } \\
n=32\end{array}$} & & \multicolumn{2}{|c|}{$\begin{array}{l}\text { Less } \\
\text { efficient } \\
\mathrm{n}=11\end{array}$} & \multicolumn{3}{|c|}{$\begin{array}{c}\text { More } \\
\text { efficient } \\
\mathrm{n}=17\end{array}$} \\
\hline & $\mathrm{n}$ & $\%$ & $\mathrm{n}$ & $\%$ & $\mathrm{p}$ & $\mathrm{n}$ & $\%$ & $\mathrm{n}$ & $\%$ & $\mathrm{p}$ \\
\hline Endomorph-ectomorph & 4 & 18.18 & 12 & 37.50 & 0.06 & 2 & 18.18 & 1 & 5.88 & 0.15 \\
\hline Ectomorphic endomorph & 4 & 18.18 & 1 & 3.13 & 0.03 & 3 & 27.27 & 0 & 0.00 & 0.01 \\
\hline Balanced endomorph & 4 & 18.18 & 1 & 3.13 & 0.03 & 0 & 0.00 & 0 & 0.00 & 0.50 \\
\hline Mesomorphic endomorph & 5 & 22.73 & 3 & 9.38 & 0.09 & 1 & 9.09 & 0 & 0.00 & 0.10 \\
\hline Mesomorph-endomorph & 1 & 4.55 & 0 & 0.00 & 0.11 & 0 & 0.00 & 0 & 0.00 & 0.50 \\
\hline Balanced ectomorph & 4 & 18.18 & 12 & 37.50 & 0.06 & 2 & 18.18 & 14 & 82.35 & 0.00 \\
\hline Central & 0 & 0.00 & 3 & 9.38 & 0.07 & 3 & 27.27 & 2 & 11.76 & 0.15 \\
\hline \multirow{3}{*}{ Somatotype category } & \multicolumn{5}{|c|}{ LIBERO } & & & & & \\
\hline & \multicolumn{2}{|c|}{$\begin{array}{l}\text { Less efficient } \\
\quad \mathrm{n}=16\end{array}$} & & $\begin{array}{c}\text { More } \\
\text { efficien } \\
n=12\end{array}$ & & & & & & \\
\hline & $\mathrm{n}$ & $\%$ & $\mathrm{n}$ & $\%$ & $\mathrm{p}$ & & & & & \\
\hline Endomorph-ectomorph & 1 & 6.25 & 1 & 8.33 & 0.42 & & & & & \\
\hline Ectomorphic endomorph & 2 & 12.50 & 0 & 0.00 & 0.10 & & & & & \\
\hline Balanced endomorph & 1 & 6.25 & 1 & 8.33 & 0.42 & & & & & \\
\hline Mesomorphic endomorph & 9 & 56.25 & 4 & 33.33 & 0.11 & & & & & \\
\hline Mesomorph-endomorph & 2 & 12.50 & 1 & 8.33 & 0.36 & & & & & \\
\hline Balanced ectomorph & 0 & 0.00 & 3 & 25.00 & 0.02 & & & & & \\
\hline Central & 1 & 6.25 & 2 & 16.67 & 0.19 & & & & & \\
\hline
\end{tabular}

Players at libero position belong to 6 somatotype categories in both subsamples. More than a half of less efficient libero players fit the mesomorphic endomorph category, and more efficient players at this position in the largest percentage fit the mesomorphic endomorph, balanced ectomorph and central somatotype categories. A significantly higher proportion of the balanced ectomorph somatotype category was identified in more efficient libero players.

In Tables 4 and 5 the significance of interpositional differences in proportions of different somatotype categories between more efficient and less efficient YFVP are shown. The most important inter-positional differences between more efficient YFVP are presented in Table 4.

Efficient setters do not differ from efficient opposite hitters and passer-hitters in any somatotype category. A significantly higher proportion of the balanced ectomorph somatotype category was identified in middle hitters, compared to all other positions. A significantly higher proportion of the mesomorphic endomorph somatotype category was identified in libero players compared to all other positions except opposite hitters. 
Table 4. Descriptive statistics of each somatotype category and significance of inter-positional differences in proportions between more efficient Croatian female volleyball players $(\mathrm{n}=96)$.

\begin{tabular}{|c|c|c|c|c|c|c|c|c|c|c|}
\hline \multirow{3}{*}{$\begin{array}{l}\text { MORE EFFICIENT } \\
\text { PLAYERS } \\
\text { Somatotype category }\end{array}$} & \multicolumn{5}{|c|}{ SETTER - OPPOSITE } & \multicolumn{5}{|c|}{ SETTER - PASSER HITTER } \\
\hline & \multicolumn{2}{|c|}{$\mathrm{n}=17$} & \multicolumn{2}{|c|}{$\mathrm{n}=17$} & \multirow[b]{2}{*}{$\mathrm{p}$} & \multicolumn{3}{|c|}{$\mathrm{n}=17$} & \multicolumn{2}{|c|}{$\mathrm{n}=32$} \\
\hline & $\mathrm{n}$ & $\%$ & $\mathrm{n}$ & $\%$ & & $\mathrm{n}$ & $\%$ & $\mathrm{n}$ & $\%$ & $p$ \\
\hline Endomorph-ectomorph & 3 & 17.65 & 3 & 17.65 & 0.50 & 3 & 17.65 & 12 & 37.50 & 0.08 \\
\hline Ectomorphic endomorph & 2 & 11.76 & 3 & 17.65 & 0.32 & 2 & 11.76 & 1 & 3.13 & 0.12 \\
\hline Balanced endomorph & 1 & 5.88 & 1 & 5.88 & 0.50 & 1 & 5.88 & 1 & 3.13 & 0.32 \\
\hline Mesomorphic endomorph & 1 & 5.88 & 2 & 11.76 & 0.27 & 1 & 5.88 & 3 & 9.38 & 0.36 \\
\hline Mesomorph-endomorph & 0 & 0.00 & 0 & 0.00 & l & 0 & 0.00 & 0 & 0.00 & l \\
\hline Balanced ectomorph & 8 & 47.06 & 8 & 47.06 & 0.50 & 8 & 47.06 & 12 & 37.50 & 0.26 \\
\hline Central & 2 & 11.76 & 0 & 0.00 & 0.07 & 2 & 11.76 & 3 & 9.38 & 0.40 \\
\hline \multirow{3}{*}{ Somatotype category } & \multicolumn{5}{|c|}{ SETTER - MIDDLE BLOCKER } & \multicolumn{5}{|c|}{ SETTER - LIBERO } \\
\hline & \multicolumn{2}{|c|}{$\mathrm{n}=17$} & \multicolumn{2}{|c|}{$\mathrm{n}=17$} & & \multicolumn{2}{|c|}{$\mathrm{n}=17$} & \multicolumn{3}{|c|}{$\mathrm{n}=12$} \\
\hline & $\mathrm{n}$ & $\%$ & $\mathrm{n}$ & $\%$ & $\mathrm{p}$ & $\mathrm{n}$ & $\%$ & $\mathrm{n}$ & $\%$ & $\mathrm{p}$ \\
\hline Endomorph-ectomorph & 3 & 17.65 & 1 & 5.88 & 0.14 & 3 & 17.65 & 1 & 8.33 & 0.24 \\
\hline Ectomorphic endomorph & 2 & 11.76 & 0 & 0.00 & 0.07 & 2 & 11.76 & 0 & 0.00 & 0.11 \\
\hline Balanced endomorph & 1 & 5.88 & 0 & 0.00 & 0.15 & 1 & 5.88 & 1 & 8.33 & 0.37 \\
\hline Mesomorphic endomorph & 1 & 5.88 & 0 & 0.00 & 0.15 & 1 & 5.88 & 4 & 33.33 & 0.03 \\
\hline Mesomorph-endomorph & 0 & 0.00 & 0 & 0.00 & I & 0 & 0.00 & 1 & 8.33 & 0.11 \\
\hline Balanced ectomorph & 8 & 47.06 & 14 & 82.35 & 0.02 & 8 & 47.06 & 3 & 25.00 & 0.11 \\
\hline Central & 2 & 11.76 & 2 & 11.76 & 0.50 & 2 & 11.76 & 2 & 16.67 & 0.35 \\
\hline \multirow[t]{2}{*}{ Somatotype category } & \multicolumn{4}{|c|}{$\begin{array}{c}\text { OPPOSITE - PASSER-HITTEF } \\
\mathrm{n}=17 \quad \mathrm{n}=32\end{array}$} & \multicolumn{6}{|c|}{$\begin{array}{cc}\text { OPPOSITE - MIDDLE BLOCKER } \\
\mathrm{n}=17\end{array}$} \\
\hline & $\mathrm{n}$ & $\%$ & $\mathrm{n}$ & $\%$ & $\mathrm{p}$ & $\mathrm{n}$ & $\%$ & $\mathrm{n}$ & $\%$ & $\mathrm{p}$ \\
\hline Endomorph-ectomorph & 3 & 17.65 & 12 & 37.50 & 0.09 & 3 & 17.65 & 1 & 5.88 & 0.14 \\
\hline Ectomorphic endomorph & 3 & 17.65 & 1 & 3.13 & 0.04 & 3 & 17.65 & 0 & 0.00 & 0.04 \\
\hline Balanced endomorph & 1 & 5.88 & 1 & 3.13 & 0.32 & 1 & 5.88 & 0 & 0.00 & 0.16 \\
\hline Mesomorphic endomorph & 2 & 11.76 & 3 & 9.38 & 0.40 & 2 & 11.76 & 0 & 0.00 & 0.07 \\
\hline Mesomorph-endomorph & 0 & 0.00 & 0 & 0.00 & I & 0 & 0.00 & 0 & 0.00 & l \\
\hline Balanced ectomorph & 8 & 47.06 & 12 & 37.50 & 0.26 & 8 & 47.06 & 14 & 82.35 & 0.02 \\
\hline Central & 0 & 0.00 & 3 & 9.38 & 0.09 & 0 & 0.00 & 2 & 11.76 & 0.07 \\
\hline
\end{tabular}

\begin{tabular}{|c|c|c|c|c|c|c|c|c|c|c|}
\hline \multirow{3}{*}{ Somatotype category } & \multicolumn{4}{|c|}{ OPPOSITE - LIBERO } & \multicolumn{6}{|c|}{ PASSER-HITTER - MIDDLE BLOCKER } \\
\hline & \multicolumn{2}{|c|}{$\mathrm{n}=17$} & \multicolumn{2}{|c|}{$\mathrm{n}=12$} & \multirow[b]{2}{*}{$\mathrm{p}$} & \multicolumn{2}{|c|}{$\mathrm{n}=32$} & \multicolumn{2}{|c|}{$\mathrm{n}=17$} & \multirow[b]{2}{*}{$\mathrm{p}$} \\
\hline & $\mathrm{n}$ & $\%$ & $\mathrm{n}$ & $\%$ & & $\mathrm{n}$ & $\%$ & $\mathrm{n}$ & $\%$ & \\
\hline Endomorph-ectomorph & 3 & 17.65 & 1 & 8.33 & 0.24 & 12 & 37.50 & 1 & 5.88 & 0.01 \\
\hline Ectomorphic endomorph & 3 & 17.65 & 0 & 0.00 & 0.06 & 1 & 3.13 & 0 & 0.00 & 0.23 \\
\hline Balanced endomorph & 1 & 5.88 & 1 & 8.33 & 0.40 & 1 & 3.13 & 0 & 0.00 & 0.23 \\
\hline Mesomorphic endomorph & 2 & 11.76 & 4 & 33.33 & 0.08 & 3 & 9.38 & 0 & 0.00 & 0.10 \\
\hline Mesomorph-endomorph & 0 & 0.00 & 1 & 8.33 & 0.11 & 0 & 0.00 & 0 & 0.00 & / \\
\hline Balanced ectomorph & 8 & 47.06 & 3 & 25.00 & 0.11 & 12 & 37.50 & 14 & 82.35 & 0.00 \\
\hline Central & 0 & 0.00 & 2 & 16.67 & 0.04 & 3 & 9.38 & 2 & 11.76 & 0.40 \\
\hline \multirow{3}{*}{ Somatotype category } & \multicolumn{4}{|c|}{ PASSER-HITTER - LIBERO } & & \multicolumn{5}{|c|}{ MIDDLE BLOCKER - LIBERO } \\
\hline & & $=32$ & & $=12$ & & \multicolumn{2}{|c|}{$\mathrm{N}=17$} & \multicolumn{3}{|c|}{$\mathrm{N}=12$} \\
\hline & $\mathrm{N}$ & $\%$ & $\mathrm{~N}$ & $\%$ & $\mathrm{p}$ & $\mathrm{N}$ & $\%$ & $\mathrm{~N}$ & $\%$ & $\mathrm{p}$ \\
\hline Endomorph-ectomorph & 12 & 37.50 & 1 & 8.33 & 0.03 & 1 & 5.88 & 1 & 8.33 & 0.40 \\
\hline Ectomorphic endomorph & 1 & 3.13 & 0 & 0.00 & 0.27 & 0 & 0.00 & 0 & 0.00 & / \\
\hline Balanced endomorph & 1 & 3.13 & 1 & 8.33 & 0.23 & 0 & 0.00 & 1 & 8.33 & 0.11 \\
\hline Mesomorphic endomorph & 3 & 9.38 & 4 & 33.33 & 0.03 & 0 & 0.00 & 4 & 33.33 & 0.01 \\
\hline Mesomorph-endomorph & 0 & 0.00 & 1 & 8.33 & 0.05 & 0 & 0.00 & 1 & 8.33 & 0.11 \\
\hline Balanced ectomorph & 12 & 37.50 & 3 & 25.00 & 0.22 & 14 & 82.35 & 3 & 25.00 & 0.00 \\
\hline Central & 3 & 9.38 & 2 & 16.67 & 0.25 & 2 & 11.76 & 2 & 16.67 & 0.35 \\
\hline
\end{tabular}

n:subject frequency, \%:relative values, p:significance of differences in proportions between less efficient and more efficient female volleyball players. 
Table 5. Descriptive statistics of each somatotype category and significance of inter-positional differences in proportions between less efficient Croatian female volleyball players $(n=85)$.

\begin{tabular}{|c|c|c|c|c|c|c|c|c|c|c|}
\hline \multirow{3}{*}{$\begin{array}{c}\text { LESS EFFICIENT } \\
\text { PLAYERS } \\
\text { Somatotype category }\end{array}$} & \multicolumn{5}{|c|}{ SETTER - OPPOSITE } & \multicolumn{5}{|c|}{ SETTER - PASSER HITTER } \\
\hline & \multicolumn{2}{|c|}{$\mathrm{n}=13$} & \multicolumn{2}{|c|}{$\mathrm{n}=24$} & \multirow[b]{2}{*}{$p$} & \multicolumn{2}{|c|}{$\mathrm{n}=13$} & \multicolumn{2}{|c|}{$\mathrm{n}=22$} & \\
\hline & $\mathrm{n}$ & $\%$ & $\mathrm{n}$ & $\%$ & & $\mathrm{n}$ & $\%$ & $\mathrm{n}$ & $\%$ & \\
\hline Endomorph-ectomorph & 0 & 0.00 & 0 & 0.00 & $\frac{T}{1}$ & 0 & 0.00 & 4 & 18.18 & 0.05 \\
\hline Ectomorphic endomorph & 1 & 7.69 & 5 & 20.83 & 0.15 & & 7.69 & & 18.18 & 0.20 \\
\hline Balanced endomorph & 2 & 15.38 & 3 & 12.50 & 0.40 & 2 & 15.38 & & 18.18 & 0.42 \\
\hline Mesomorphic endomorph & 5 & 38.46 & 10 & 41.67 & 3 & 5 & 38.46 & & 22.73 & 0.16 \\
\hline Mesomorph-endomorph & 2 & 15.38 & 1 & 4.17 & 0.12 & 2 & 15.38 & & 4.55 & 0.13 \\
\hline Balanced ectomorph & 1 & 7.69 & 2 & 8.33 & 0.47 & 1 & 7.69 & 4 & 18.18 & 0.20 \\
\hline Central & 2 & 15.38 & 3 & 12.50 & 0.40 & 2 & 15.38 & 0 & 0.00 & 0.03 \\
\hline \multirow{3}{*}{ Somatotype category } & \multicolumn{5}{|c|}{ SETTER - MIDDLE BLOCKER } & \multicolumn{5}{|c|}{ SETTER - LIBERO } \\
\hline & \multicolumn{2}{|c|}{$\mathrm{n}=13$} & \multicolumn{2}{|c|}{$\mathrm{n}=11$} & & \multicolumn{2}{|c|}{$\mathrm{n}=13$} & \multicolumn{2}{|c|}{$\mathrm{n}=16$} & \\
\hline & $\mathrm{n}$ & $\%$ & $\mathrm{n}$ & $\%$ & $\mathrm{p}$ & $\mathrm{n}$ & $\%$ & $\mathrm{n}$ & $\%$ & $\mathrm{p}$ \\
\hline Endomorph-ectomorph & 0 & 0.00 & 2 & 18.18 & 0.05 & 0 & 0.00 & & 6.25 & 0.18 \\
\hline Ectomorphic & 1 & 7.69 & 3 & 27.27 & 0.10 & & 7.69 & & 12.50 & 0.37 \\
\hline Balanced e & 2 & 15.38 & 0 & 0.00 & 0.09 & & 15.38 & & 6.25 & 0.22 \\
\hline Mesomor & 5 & 38.46 & 1 & 9.09 & 0.05 & & 38.46 & & 56.25 & 0.17 \\
\hline Mesomol & 2 & 15.38 & 0 & 0.00 & 0.09 & 2 & 15.38 & & 12.50 & 0.42 \\
\hline Balanced $€$ & 1 & 7.69 & 2 & 18.18 & 0.29 & 1 & 7.69 & & 0.00 & 0.13 \\
\hline Central & 2 & 15.38 & 3 & 27.27 & 0.24 & 2 & 15.38 & 1 & 6.25 & 0.22 \\
\hline \multirow{3}{*}{ Somatotype category } & \multicolumn{5}{|c|}{ OPPOSITE - PASSER-HITTER } & \multicolumn{5}{|c|}{ OPPOSITE - MIDDLE BLOCKER } \\
\hline & & $=24$ & & $=22$ & & & 24 & & 11 & \\
\hline & $\mathrm{n}$ & $\%$ & $\mathrm{n}$ & $\%$ & $\mathrm{p}$ & $\mathrm{n}$ & $\%$ & $\mathrm{n}$ & $\%$ & $\mathrm{p}$ \\
\hline Endomorph-ectomorph & 0 & 0.00 & 4 & 18.18 & 0.01 & 0 & 0.00 & 2 & 18.18 & 0.02 \\
\hline Ectomorphic endomorph & 5 & 20.83 & 4 & 18.18 & 0.41 & 5 & 20.83 & & 27.27 & 0.34 \\
\hline Balanced end & 3 & 12.50 & 4 & 18.18 & 0.30 & 3 & 12.50 & & 0.00 & 0.11 \\
\hline Mesomorphic e & 10 & 41.67 & 5 & 22.73 & 0.09 & 10 & 41.67 & & 9.09 & 0.03 \\
\hline esomorph-c & 1 & 4.17 & 1 & 4.55 & 0.48 & 1 & 4.17 & 0 & 0.00 & 0.25 \\
\hline Balanced & 2 & 8.33 & 4 & 18.18 & 0.16 & 2 & 8.33 & 2 & 18.18 & 0.20 \\
\hline Cen & 3 & 12.50 & 0 & 0.00 & 0.04 & 3 & 12.50 & 3 & 27.27 & 0.14 \\
\hline & & OSITE & $\overline{\mathrm{IB}}$ & & PASS & & $\mathrm{ER}-\mathrm{M}$ & & BLOC & ER \\
\hline Somatotype category & & & & & & & & & & \\
\hline & $\mathrm{n}$ & $\%$ & $\mathrm{n}$ & $\%$ & $\mathrm{p}$ & $\mathrm{n}$ & $\%$ & $\mathrm{n}$ & $\%$ & $\mathrm{p}$ \\
\hline Endomorph- & 0 & 0.00 & 1 & 6.25 & 0.11 & 4 & 18.18 & & 18.18 & 0.50 \\
\hline Ectomorphic & 5 & 20.83 & 2 & 12.50 & & 4 & 18.18 & & 27.27 & 0.27 \\
\hline & 3 & .50 & 1 & 6.25 & & 4 & 18.18 & U & 0.00 & 0.07 \\
\hline Mesomorphic & 10 & 41.67 & 9 & 25 & & 5 & 22.73 & 1 & 9.09 & 0.17 \\
\hline somorph-e & 1 & 17 & 2 & 12.50 & & 1 & 4.55 & U & 0.00 & 0.24 \\
\hline Balanced & 2 & 8.33 & 0 & 0.00 & & 4 & 18.18 & 2 & 18.18 & 0.50 \\
\hline & 3 & 12.50 & 1 & 6.25 & 0.26 & 0 & 0.00 & 3 & 27.27 & 0.01 \\
\hline & & SSER-H & & LIBE & & & LE BI & & - LIBE & \\
\hline Somatotype category & & & & & & & & & $\mathrm{n}=16$ & \\
\hline & $\mathrm{n}$ & $\%$ & $\mathrm{n}$ & $\%$ & $\mathrm{p}$ & $\mathrm{n}$ & $\%$ & $\mathrm{n}$ & $\%$ & $\mathrm{p}$ \\
\hline Endomorph-e & 4 & 18.18 & 1 & 6.25 & 0.14 & 2 & 18.18 & 1 & 6.25 & 0.17 \\
\hline Ectomorphic endomorph & 4 & 18.18 & 2 & 12.50 & 32 & 3 & 27.27 & 2 & 12.50 & 0.17 \\
\hline alanced ens & 4 & 18.18 & 1 & 6.25 & 14 & 0 & 0.00 & 1 & 6.25 & 0.20 \\
\hline Mesomorphic en & 5 & 22.73 & 9 & 56.25 & 02 & 1 & 9.09 & 9 & 56.25 & 0.01 \\
\hline Mesomorph-endomorph & 1 & 4.55 & 2 & 12.50 & 0.20 & 0 & 0.00 & 2 & 12.50 & 0.11 \\
\hline Balanced ectomorph & 4 & 18.18 & 0 & 0.00 & 0.04 & 2 & 18.18 & 0 & 0.00 & 0.04 \\
\hline Central & 0 & 0.00 & 1 & 6.25 & 0.12 & 3 & 27.27 & 1 & 6.25 & 0.07 \\
\hline
\end{tabular}

n:subject frequency, \%:relative values, p:significance of differences in proportions between less efficient and more efficient female volleyball players. 
In a subsample of less efficient players (Table $5)$ it can be noticed that there were no differences between setters, opposite hitters and liberos. The highest number of significant differences in proportions of each somatotype category was obtained by comparing the players at those positions with passer-hitters and middle blockers. In less efficient setters, opposite hitters and liberos the mesomorphic endomorph somatotype category has a significantly higher frequency, whereas in less efficient passer-hitters and middle blockers the endomorph-ectomorph category was significantly more frequent.

\section{DISCUSSION}

A greater number of YFVP at passer-hitter, middle hitter and setter positions belongs to the more efficient players. Therefore, it can be assumed that in the U15 age group players at these positions contribute more to team success. In volleyball, setters organize almost every attack during play, so it is important to have quality players at this position. Passer-hitters in the U-15 age group usually spike most balls during the game and score most points, which confirms the importance of this position. In a competitive sense, the middle hitter position is not much expressed at this age group, partially due to a relatively low frequency of the first tempo attack, and partially due to the demanding technique of blocking, which is perfected only by a small number of players of this age group. That is why a greater number of more efficient players at middle hitter position are probably a consequence of the fact that at this position there are usually more players among starters (2) than among non-starters (1). Opposite hitter and libero position are not yet sufficiently profiled in this age group considering that at these positions players mostly belong to the less efficient group. YFVP are not tall enough, do not have sufficient jumping and upper body power for powerful attacking against the solid block and from the second row, which is an important characteristic of opposite players in senior competition. That is why in practice more efficient attackers in this age group are assigned the role of passer-hitters. Libero position is not used in the U-15 age group as much as in junior and senior age groups because coaches want all players to be versatile, which is not possible if some of them do not play field defense and are constantly substituted by libero players. That is probably why most players at this position do not belong to the group of more efficient players.

The dominance of the endomorph somatotype component on the overall sample (Table 1) was expected considering the characteristics of the sample consisting of YFVP of lower (club) quality level. In somatotype studies that were done on similar samples, female volleyball players were also more endomorph than ectomorph. For example, Soarez and De Paula (21) determined the mean somatotype of 4.9-2.42.9 among young Brazilian female volleyball players of the club quality level, and mean somatotype of young non-elite Spanish female players (19) was 4.6-5.3-2.8. In some previous studies involving a sample of young female Croatian volleyball players (e.g., 32) excess subcutaneous fat tissue was also found in young female volleyball players of the club quality level, which also indirectly indicates the dominance of the endomorph somatotype component.

However, in investigations of YFVP at the national team quality level the ectomorph somatotype component was expressed the most. For example, mean somatotype of young Brazilian female national team members (18) was 3.1-2.2-3.9, whereas that of young Turkish national team members (1) was 3.4-2.1-4.5.

The results of the aforementioned studies, which indicate that the ectomorph somatotype component is more prominent in more efficient young female volleyball players, whereas the endomorph component is more prominent in less efficient players, are congruent with the most important findings of the current study.

The most important contribution of this study is that somatotype categories within each position (Table 3) are being analysed for the first time, and separately on subsamples of more efficient and less efficient players. As on the overall sample, a significantly higher frequency of somatotype categories predominated by the ectomorph somatotype component was also found on the subsample of more efficient players. A significantly higher frequency of somatotype categories predominated by the endomorph somatotype component was found on the subsample of less efficient YFVP. 
Some authors $(37,38)$ claim that the somatotypes of top young female athletes do not substantially differ from the respective top adult athletes' somatotype. Thus, it can be concluded that the exact type of body build is important for success in all player positions in volleyball, both in younger age groups and in senior competition. Considering that balanced ectomorph is the most frequent somatotype category in more successful players in all positions, this is probably the type of body build that contributes to playing efficacy. Similar conclusions are put forth by Malousaris et al. (23), who point out that mesomorphy used to be the primary component of competitive female volleyball players' somatotype in the last two decades, but recent studies indicated a trend toward ectomorphy. Ectomorph somatotype component is mostly genetically determined. That is why in the process of identification and selection for each player position (and especially the middle hitter position), players who, in addition to other predispositions, also have this prominent somatotype component should be selected. On the other hand, high percentage of YFVP in the subsample of less efficient players is characterized by domination of the endomorph component, which is the least genetically determined (39). This somatotype component should be reduced by proper nutrition and appropriate training load. As volleyball is not a contact sport, it is important to develop strength by methods that would not lead to excessive muscle mass increase, and therefore the mesomorph somatotype component would be maintained at the required level.

By analysing each somatotype category according to position, it can be noticed that only 7 out of the possible 13 somatotype categories were obtained on a relatively large sample of 181 young female volleyball players. It is indicative that there was no somatotype category dominated by the mesomorph somatotype component. This is probably only partly due to the previously mentioned specificity of volleyball as a non-contact sport, because both gender and age specificities may contribute to such results. It is known that the muscle mass increase mostly occurs in the period during and after puberty, and that women are characterized by less prominent muscle mass in comparison to men (16,22). Previously conducted phases of detection and identification of volleyball talents within clubs can also contribute to sample homogenization according to the criterion of the number of somatotype categories.

The smallest number of somatotype categories (only three) was obtained in the subsample of more successful middle hitters. Over $80 \%$ of players at this position belong to the balanced ectomorph somatotype category. This indirectly indicates that, according to body build, the most stringent selection in practice was conducted exactly for that position.

Body height and arm length, that are above average (as witnessed by, i.e., standing reach), are very important for efficient play at this position. Such body build enables middle hitters to reach the necessary height above the net with their arms quickly. This way they can spike the first tempo attacks in time, but more importantly, successfully block fast and diverse opponents' attacks.

Inter-positional differences were analysed in this study separately for the subsample of more efficient (Table 4) and the subsample of less efficient young female volleyball players (Table 5). The authors assumed that coaches at the club level pay more attention to the selection of quality players for specific positions, and assign positions to less efficient players more according to the criterion of current necessity for filling empty places in the team, and not according to their player predispositions for certain positions. Based on the obtained inter-positional differences, it can be concluded that assumption has not been confirmed, because an equal, relatively small number of significant interpositional differences was found in proportions of the somatotype categories in both subsamples.

Small inter-positional differences in both subsamples of YFVP are probably the consequence of the previous selection process in clubs. Many girls in Croatia train volleyball in clubs (in larger cities even several hundreds in one club). Therefore, even before YFVP are selected for certain player positions, a fairly rigorous selection process is performed. In that process, from an extremely large sample of girls who train volleyball in a club, only 15-20 are selected to become members of the competitive team in their age group. During selection 
coaches also consider suitable body build, i.e., give priority to those players, who are characterized by prominent ectomorph somatotype component. Given that in previous selection body build was an important criterion for including a player in the competitive team, in the following phase of selection for specific player positions coaches probably take some other factors into consideration, e.g., motor abilities, technique and tactics about certain volleyball elements, etc. This mostly refers to the libero position, in which body build probably makes the smallest contribution to performance quality, and least to the middle hitter position, which requires prominent ectomorphy of the somatotype.

\section{CONCLUSION}

This study is the first to investigate both interpositional and intra-positional differences in somatotype categories of young female volleyball players. The main findings indicate that there was a significantly higher frequency of somatotype categories with a predominant ectomorph component in all player positions in a subsample of more efficient players, and there was a significantly higher frequency of somatotype categories predominated by the endomorph component in a subsample of less efficient players. Contrary to the authors' expectations, there were no prominent inter-positional differences found in the subsample of more efficient YFVP in comparison to inter-positional differences in the subsample of less efficient YFVP.

\section{ACKNOWLEDGEMENT}

The authors wish to thank all the athletes and coaches who volunteered within this study. This work has been supported by the Croatian Science Foundation under the project number [6524].

\section{APPLICABLE REMARKS}

- The results obtained in this study provide a clearer insight into interpositional and intra-positional differences in somatotype categories of young female volleyball players:

a) The obtained intra-positional differences between less efficient and more efficient YFVP in proportions of each somatotype category indicate that with the increase of efficacy at all positions in volleyball a trend toward ectomorphy can be noticed.

b) Considering a high level of innateness of that somatotype component in the process of talent identification for volleyball, suitable body build should also be one of the important criteria.

c) In the training process great attention should also be paid to the reduction of subcutaneous fat tissue.

d) Small inter-positional differences in frequency of each somatotype category indicate a previous homogeneity of samples in terms of body build in the first phases of selection.

e) Therefore, in the selection process for specific positions body build should not be the primary selection criterion.

f) The middle hitter position is certainly the exception, which requires specific body build predominated by the ectomorph somatotype component as a necessary precondition for success.

- Using the results presented here, volleyball coaches will be able to better detect talents for volleyball, and also to assign the most appropriate player positions according to their body build.

\section{REFERENCES}

1. Ayan V, Bektas Y, Ali Emre E. Anthropometric a performance characteristics of Turkey National U-14 volleyball players. African Journal for Physical, Health Education, Recreation \& Dance. 2012;18(2):395-403.

2. Bandyopadhyay A. Anthropometry and body composition in soccer and volleyball players in West Bengal, India. Journal of physiological anthropology. 2007;26(4):501-505.

3. Bayios IA, Bergeles NK, Apostolidis NG, Noutsos KS, Koskolou MB. Anthropometric, body composition and somatotype differences of Greek elite female basketball, volleyball and handball players. Journal of sports medicine and physical fitness. 2006;46(2):271-280. 
4. Buśko K, Lewandowska J, Lipińska M, Michalski R, Pastuszak A. Somatotype-variables related to muscle torque and power output in female volleyball players. Acta of Bioengineering and Biomechanis. 2013;15(2):119-126.

5. Cabral BG, Cabral SA, Toledo IV, Dantas PM, Miranda HF, Knakcfuss MI. Anthropometry and somatotype: determining factors in the selection of athletes in Brazilian volleyball. Revista Brasileira de Ciências do Esporte. 2011;33(3):733-746.

6. Carvajal W, Betancourt H, León S, Deturnel Y, Martínez M, Echevarría I, Castillo ME, Serviat N. Kinanthropometric profile of Cuban women Olympic volleyball champions. MEDICC Review. 2012;14(2):1622.

7. Fonseca-Toledo C, Roquetti P, Fernandes-Filho J. [Anthropometrical profile of Brazilian junior volleyball players for different sports requirement levels]. Revista de Salud Pública. 2010;12(6):915-928.

8. Gabbett T, Georgieff B. Physiological and anthropometric characteristics of junior national, state, and novice volleyball players. Journal of Strength \& Conditioning Research. 2007;21(3):902-908.

9. Gabbett T, Georgieff B, Domrow N. The use of physiological, anthropometric, skill data to predict selection in a talent-identified junior volleyball squad. Journal of sports sciences. 2007;25(12):1337-1344.

10. Gaurav V, Singh M, Singh S. Anthropometric characteristics, somatotyping and body composition of volleyball and basketball players. Journal of Physical Education and Sports Management. 2010;1(3):28-32.

11. Martín-Matillas M, Valadés D, Hernández-Hernández E, Olea-Serrano F, Sjöström M, Delgado-Fernández M, Ortega FB. Anthropometric, body composition and somatotype characteristics of elite female volleyball players from the highest Spanish league. Journal of sports sciences. 2014;32(2):137-148.

12. Milić M, Grgantov Z, Katic R. Somatotype of young female volleyball players. Exercise and Quality of Life. 2012;4(2):7-15.

13. Nikolaidis PT, Ziv G, Arnon M, Lidor R. Physical characteristics and physiological attributes of female volleyball players--the need for individual data. Journal of Strength \& Conditioning Research. 2012;26(9):25472557.

14. Silva S, Maia J. Analysis of the aspects related with the performance of motor skills female volleyball players of 12 to 14 years. In: Mesquita I, Moutinho C, Faria R, eds. Investigação em voleibol: estudos ibéricos. Saúde e Sá; 2003. p. 220.

15. Tsunawake N, Tahara Y, Moji K, Muraki S, Minowa K, Yukawa K. Body composition and physical fitness of female volleyball and basketball players of the Japan inter-high school championship teams. Journal of physiological anthropology and applied human science. 2003;22(4):195-201.

16. Viviani F, Baldin F. The somatotype of «amateur» Italian female volleyball-players. The Journal of sports medicine and physical fitness. 1993;33(4):400-404.

17. Zhang Y. An investigation on the anthropometry profile and its relationship with physical performance of elite Chinese women volleyball players. M.Sc. thesis, Southern Cross University; 2010 [cited 20178 February]; Available from: http://epubs.scu.edu.au/cgi/viewcontent.cgi?article=1192\&context=theses.

18. Cabral BG, Cabral SA, Batista GR, Fernandes Filho J, Knackfuss MI. Somatotype and anthropometry in brazilian national volleyball teams. Motricidade. 2008;4:67-73.

19. Hoyo Lora MD, Sañudo B, Carrasco L. [Assessment of somatotype in young volleyball players: validity as criteria to select young sports talents]. Rev Bras Cineantropom Desempenho Hum. 2008;10(3):255-260.

20. Riegerová J, Ryšavý J. Somatodiagnostics of female, secondary school age volleyball players. Acta Universitatis Palackianae Olomucensis Gymnica. 2001;31(1):37-42.

21. Soares CA, De Paula AH. [Analysis of kinanthropometric profile of young volleyball players aged 12 to 15 years]. Movimentum-Revista Digital de Educação Física. 2006;1:1-15.

22. Gualdi-Russo E, Zaccagni R. Somatotype, role and performance in elite volleyball players. Journal of Sports Medicine and Physical Fitness. 2001;41(2):256-262.

23. Malousaris GG, Bergeles KBNK, Barzouka KG, Bayios IA, Nassis GP, Koskolou MD. Somatotype, size and body composition of competitive female volleyball players. Journal of Science and Medicine in Sport. 2008;11(3):337-344.

24. Pelin C, Kürkçüoğlu A, Ozener B, Yazici AC. Anthropometric characteristics of young Turkish male athletes. Collegium antropologicum. 2009;33(4):1057-1063.

25. Hopkins WG, Marshall SW, Batterham AM, Hanin J. Progressive statistics for studies in sports medicine and exercise science. Medicine+ Science in Sports+ Exercise. 2009;41(1):3-13.

26. Carter JEL. Somatotyping. In: Norton KT, Olds T, eds. Anthropometrica 6. University of New South Wales Press; 1996. p. 147-170.

27. Carter JEL. The Heath-Carter anthropometric somatotype - instruction manual. Department of Exercise and Nutritional Sciences, San Diego State University; 2002. 
28. Carter JEL, Heath BH. Somatotyping: development and applications. Cambridge University Press; 1990.

29. Heath B, Carter JEL. A modified somatotype method. American Journal of physical anthropology. 1967;27(1):57-74.

30. Kinanthropometry IS. International standards for anthropometric assessment. Underdale, SA, Australia: ISAK. 2001:1-33.

31. Goulding M. Somatotype - 1.2.5. Calculation and analysis. Sweat Technologies; 2010.

32. Grgantov Z, Katic R, Jankovic V. Morphological characteristics, technical and situation efficacy of young female volleyball players. Collegium antropologicum. 2006;30(1):87-96.

33. Grgantov Z, Milić M, Katic R. Identification of explosive power factors as predictors of player quality in young female volleyball players. Collegium antropologicum. 2013;37(2):61-68.

34. Milić M, Grgantov Z, Katic R. Biomotor status and kinesiological education of girls aged 10 to 12 yearsexample: volleyball. Collegium antropologicum. 2012;36(3):959-966.

35. Marfell-Jones MJ, Stewart AD, de Ridder JH. International standards for anthropometric assessment. 2012.

36. Duquet W, Carter JEL. Somatotyping. In: Eston R, Reilly T, eds. Kinanthropometry and exercise physiology laboratory manual: tests, procedures and data. Anthropometry 1. E \& FN Spon; 2001. p. 54-72.

37. Malina RM, Shoup RF. Anthropometric and physique characteristics of female volleyball players at three competitive levels. Humanbiol Budapest. 1985;16:105-116.

38. Papadopoulou DS, Gallos KG, Paraskevas G. The somatotype of Greek female volleyball athletes. Int J Volleyball Res. 2002;5(1):22-25.

39. Peeters MW, Thomis MAI, Beunen GP, Malina RM. Genetics and sports: an overview of the pre-molecular biology era. Medicine and Sport Science. 2009;54:28-42. 